\title{
Response to Commentary on The Trauma Treatment in Young Children with Developmental Disabilities: Applications of the Child-Parent Psychotherapy (CPP) Model to the Cases of "James" and "Juan"
}

\section{Child-Parent Psychotherapy in the Context of the Developmental Disability and Medical Service Systems}

\section{MARIAN E. WILLIAMS, ${ }^{\text {,c }}$ MELISSA C. CARSON, ${ }^{\mathrm{a}}$ IRINA ZAMORA, ELIZA K. HARLEY, ${ }^{b}$ \& PATRICIA P. LAKATOS ${ }^{b}$}

\author{
${ }^{\mathrm{a}}$ Keck School of Medicine of USC, University of Southern California \\ bUSC University Center for Excellence in Developmental Disabilities at Children’s Hospital Los Angeles \\ ${ }^{\mathrm{C}}$ Correspondence regarding this article should be addressed to Marian E. Williams, USC University Center for \\ Excellence in Developmental Disabilities, Children's Hospital Los Angeles, 4650 Sunset Blvd., MS\#53, Los \\ Angeles, CA 90027. \\ Email: mwilliams@chla.usc.edu \\ Note: Eliza K. Harley is now at Rady Children’s Hospital San Diego.
}

\begin{abstract}
Child-Parent Psychotherapy (CPP), an evidence-based dyadic treatment for young children who have experienced trauma, shows promise in intervening with children with developmental disabilities, as illustrated in the cases we described of 14-month-old "James" and 6-year-2month-old "Juan" (Harley, Williams, Zamora, \& Lakatos, 2014). In their thoughtful commentary on these cases, Ghosh Ippen, Noroña, \& Lieberman (2014) discuss additional clinical considerations important to the use of CPP with this population. In this response, we explore further implementation of CPP in the context of the developmental disability and medical service systems. In particular, we highlight the importance of resilience in parents' responses to their child's developmental disability, contributions from pediatric psychology to understanding pediatric medical traumatic stress, infusing relationship-based approaches in collaboration with other service systems, and addressing barriers to service access in ethnic minority families. Finally, we provide recommendations for training and fidelity monitoring when applying CPP to children with developmental disabilities and their families.
\end{abstract}

Key Words: pediatric medical traumatic stress; developmental disability; trauma-informed care; infant mental health; case studies; clinical case studies

In their insightful and rich commentary, Ghosh Ippen, Noroña, \& Lieberman (2014) provide important clinical perspectives on the application of Child-Parent Psychotherapy (CPP) to young children with developmental disabilities who have experienced trauma. Their commentary explores the roots of the CPP model in infant-parent psychotherapy with children with disabilities; ways in which the parent-child relationship may be impacted by developmental 
disability; research about the co-occurrence of trauma and developmental disability; and ways in which the CPP therapist can work in partnership with the early intervention system to meet the unique needs of young children with developmental disabilities. Finally, they share important insights about the intersection of culture and disability. In this response, we explore in more depth some of the critical issues raised in Ghosh Ippen et al.'s (2014) commentary, with a focus on ways in which the CPP therapist incorporates a comprehensive understanding of and collaboration with service systems when working with young children with developmental disabilities and/or medical trauma.

\section{RESILIENCE AND TRANSFORMATION IN FAMILIES OF CHILDREN WITH DISABILITIES}

Ghosh Ippen et al (2014) address the importance of considering risk and protective factors within the parent-child relationship, with a particular focus on factors unique to families who have a young child with a developmental disability. They summarize literature documenting parental responses to learning that a child has a developmental disability, including stress, feelings of grief and shock, symptoms of depression, and post-traumatic responses. In addition, they highlight ways in which CPP provides an opportunity to intervene early in the attachment relationship to promote a resolution of the parent-child relationship and help the parent to make meaning of the experience of disability.

While acknowledging the profound challenges that developmental disability may present to family relationships, an emerging literature focusing on family resilience and the potentially transformative impact of having a child with a disability provides an important additional perspective. Research using surveys and qualitative interviews with families of a child with a disability has revealed that the majority of parents does not experience lasting distress and in fact reports positive outcomes of having a child with a disability on their families and on themselves (e.g., Bayat, 2007; Emerson, 2003; Scorgie \& Sobsey, 2000). Most families interviewed, while acknowledging difficulties, also identified positive transformations that arose from having a child with a disability. These included increased sensitivity to and tolerance of individuals who are different, increased communication and cohesion in the family, a greater sense of what matters in life or of spiritual meaning, and increased confidence and skills related to navigating the service system and managing other challenges (e.g., Green, 2007; Scorgie \& Wilgash, 2008; Taunt \& Hastings, 2002).

Further, research suggests that subjective burden (e.g. emotional distress) is less impactful on families than objective burdens such as needing to advocate for services, the time and expense of accessing services and attending appointments, and lack of support from employers for time off work to attend to the needs of the child with a disability (Green, 2007). In Green's study of 81 mothers of children with disabilities, perceived stigma related to disability was associated with increased reports of objective burden. While Ferguson (2002) noted positive changes in the disability field as greater resources and rights for individuals with disability have been established, a study by Emerson (2003) found that economic disadvantage moderates the impact of disability on maternal wellbeing. Thus, families living in poverty are likely to experience greater objective burden than more economically advantaged families, and these 
factors related to disadvantage may impact family resilience more than factors such as the child's level of disability.

These perspectives on resilience in families of children with developmental disabilities have implications for clinical practice and for the training of medical providers and therapists. First, it is critical to imbed our thinking about developmental disability within a societal context and to recognize the profound impact of a family's economic circumstances, the availability of resources, and the presence of stigma about individuals with disability on the family's perceptions of disability and of burden. For example, in the case of 14-month-old James, who had a stroke following surgery and was presented in our case study (Harley, Williams, Zamora, \& Lakatos (2014), we encounter a family of five living in one room and needing to accommodate the presence of a nurse and therapists within the confined space where they are caring for their child with special healthcare needs as well as their two older children. The family's experience of poverty and disadvantaged access to resources is essential to understanding their experience and perceptions of their infant's changed functioning and needs.

Second, learning about family narratives of resilience, transformation, and meaningmaking are key components of the education of professionals preparing to work in the field of developmental disability. These narratives may lead medical professionals to a different perspective when talking with a family about their child with a disability, one that is infused with hope and pride and not only with sadness and sympathy. Parents vividly remember the first time a professional talks to them about their child's disability or diagnosis and have strong feelings about the experience (e.g., Chamak, Bonniau, Oudaya, \& Ehrenberg, 2011; Hedov, Wikblad, \& Anneren, 2007; O’Brien \& Daggett, 2006). The professionals’ own perceptions about the meaning of disability and about the quality of life and inherent value of individuals with disabilities powerfully influence their manner and choice of words when communicating with families and when training new professionals (Shakespeare, Iezzoni, \& Groce, 2009; Stanton \& Besser, 1998).

Finally, researchers who focus on transformation and resilience have delineated life management strategies that may help guide interventions with families who are experiencing challenges. For example, Scorgie and Wilgosh (2008) describe essential strategies found to be helpful to parents of children with disabilities, including making meaning and developing a positive reframing of their situation, maintaining a balance in their roles and responsibilities for their child and in their personal and family life, and learning to locate and utilize resources effectively. Scorgie, Wilgosh, and Sobsey (2004) note the importance of emphasizing family strengths, recognizing and asking about positive or beneficial effects of the family's experience with their child, and remaining aware of the range of common outcomes for families who have a child with a disability, including positive transformation.

Ghosh Ippen et al. (2014) note the variation in parents' responses to a child's disability and the influence of factors such as the timing of diagnosis, available sources of support, and resources. An implication for CPP with this population is the importance of supporting families in learning to navigate the service system and identify available resources. In addition, linking families with other parents who have a child with a disability may provide essential support and practical assistance. Family resource centers, parent support groups, and parent advocacy 
organizations provide parents with opportunities to get to know families who have children with disabilities at different ages and stages of life, learn about resources and strategies to access them, increase social support, and discover opportunities to support others (Kerr \& McIntosh, 2000; Law, King, Stewart, \& King, 2001; Solomon, Pistrang, \& Barker, 2001). A survey of 1,005 caregivers of children with autism found that parents from lower income and minority families were significantly less likely to participate in autism-specific support groups than more affluent and white families (Mandell \& Salzer, 2007). Ethnic-specific parent organizations may help to bridge this gap, although challenges related to work demands, lack of transportation, or lack of child care may limit access to such resources for low income families. Mandell and Salzer (2007) also found that only a small minority of families had been referred to parent support groups by their provider; most found the groups on their own. The CPP therapist can provide an important bridge by linking families of children with disabilities to other family supports which can extend beyond the time period and role limitations of the therapeutic relationship.

\section{PEDIATRIC MEDICAL TRAUMATIC STRESS AND CPP}

The other case we presented in our case study was James, a 14-month-old who had experienced medical trauma (surgery and stroke) and who was manifesting attachment problems. As illustrated in this case, often families must cope with ongoing medical stressors in addition to the diagnosis of a developmental disability in their infant. Not only is there significant comorbidity between developmental disabilities and trauma exposure, as noted by Ghosh Ippen et al. (2014), but there are inherent factors related to a child being diagnosed with a medical condition and being exposed to a pediatric medical setting that may contribute to a child or family experiencing traumatic stress reactions. Our experience has indicated that CPP is appropriate for those young children and families whose traumatic event is pediatric medical traumatic stress.

The Center for Pediatric Traumatic Stress (n.d.) defines pediatric medical traumatic stress (PMTS) as "a set of psychological and physiological responses of children and their families to pain, injury, serious illness, medical procedures, and invasive or frightening treatment experiences.” Many pediatric patients and their families experience some degree of acute traumatic stress following illness, hospitalization, or painful medical procedures, and some will develop full symptoms of posttraumatic stress disorder (PTSD) (Kassam-Adams \& Fein, 2003; Saxe, Vanderbilt, \& Zuckerman, 2003; Stuber, Shemesh, \& Saxe, 2003). Viewing a pediatric medical diagnosis and subsequent required medical treatment through a pediatric medical traumatic stress framework helps identify opportunities for supporting families and children through their experiences, including children in the birth to 5 year age range, and preventing more serious symptoms from developing. Responses to pediatric medical traumatic stress are related to the person's subjective experience and may include "symptoms of arousal, reexperiencing or avoidance, or a constellation of these symptoms such as posttraumatic stress disorder” (Center for Pediatric Traumatic Stress, n.d.)

Kazak, Kassam-Adams, Schneider, Zelikovsky, and Alderfer \& Rourke (2006) proposed a three-phase model of pediatric medical traumatic stress. The first phase, peritrauma, is the 
period during and immediately following a potentially traumatic medical event. Phase two consists of early, ongoing, and evolving responses, during which the family has to face the ongoing demands and challenges associated with the medical diagnosis and treatment. Phase three refers to longer term pediatric medical traumatic stress which considers the long term reactions to a medical event or condition, with the understanding that traumatic responses do not necessarily subside over time and long-term traumatic reactions may occur separately from the acute medical treatment that occurred. This model moves along the continuum from prevention to intervention and emphasizes a family perspective of medical events, a perspective that is particularly important when thinking of young children who rely on their parents for their sense of safety and wellbeing and which is core to CPP.

When considering James' case from a CPP perspective, assessment of the parent's life stressors was important in order to identify how their own trauma histories may impact their reactions and ability to support James during triggering events in the medical setting. Due to the constraints of an inpatient medical setting and the acute, ongoing medical care in that milieu, one can imagine barriers to completion of additional assessment instruments within that setting, and in the midst of a family experiencing the ongoing medical crisis of their child. However, it is important to keep in mind that when providing CPP intervention, assessment of the caregiver's trauma history is important and should be completed as soon as possible. In some cases, it may make sense to provide therapeutic intervention to assist in stabilization and emotional regulation while the child is hospitalized utilizing a CPP-informed approach. This may consist of working to engage the family and beginning to establish a trauma framework. Then, following discharge, CPP intervention would begin.

In the field of pediatric psychology, protective and risk factors have been identified to help guide assessment and intervention for children and families who might be at risk for more severe traumatic stress reactions following pediatric medical events. The following are salient protective factors impacting the responses of young children and families: parental presence around the time of the illness, injury, or medical treatment; parental support; and maintaining or re-establishing age-appropriate social activities (National Child Traumatic Stress Network, 2004). CPP works toward reducing the potential impact of future stressful medical experiences by increasing parental support and parental presence through a focus on strengthening the parentchild dyadic relationship and through increasing the emotional availability of the parent to respond to the child's cues and make meaning of the child's experience and behavior in the medical setting. Additionally, the following risk factors have been identified as possibly contributing to a more severe traumatic stress reaction in situations of pediatric medical traumatic stress: pre-existing anxiety and/or other psychological difficulties; history of trauma exposure or previous traumatic stress symptoms; severe acute traumatic symptoms that occur within a short period of time surrounding the medical event; length of hospitalization; hospitalization within an intensive care unit; separation from parents and separation anxiety during emergent care or during the hospitalization; perceptions during treatment; extreme avoidance of things that are reminders of a traumatic event; and social and emotional withdrawal. These protective and risk factors are all areas that would potentially be assessed during the Assessment and Engagement phase of CPP. 
When a young child is diagnosed with a chronic medical condition, the potential trauma begins at the time of diagnosis and the traumatic events may be ongoing, in the form of painful, recurrent medical procedures that the child and family must endure in order to optimize the child's health outcomes. Research conducted within the field of pediatric psychology also documents the negative impact on many caregivers. Landolt and colleagues (Landolt, Vollrath, Ribi, Gnehm \& Sennhauser, 2003) found that in a sample of parents whose child had been involved in an accident, received a new diagnosis of cancer, or received a new diagnosis of diabetes mellitus, $16 \%$ of fathers and $24 \%$ of mothers met full diagnostic criteria for PTSD, with up to $44 \%$ of parents of children with a new diagnosis of cancer meeting criteria for PTSD. Parents of childhood cancer survivors and parents of solid organ transplant recipients have also exhibited posttraumatic stress symptoms (Kazak, Stuber, Barakat, Meeske, Guthrie \& Meadows, 1998; Young, Mintzer, Seacord, Castañeda, Mesrkhani \& Stuber, 2003). Parental perception of the outcome of transplant surgery may impact their level of post traumatic stress symptoms (Young et al., 2003). The emphasis in CPP on assessing the parent's trauma history, including the parent's experience of a child's initial medical and/or developmental disability diagnosis and the parents' perception of subsequent medical intervention, is essential in understanding a parent's capacity for emotional regulation and dyadic regulation during future medical intervention. This assessment also helps the therapist and parent understand potential trauma triggers within the medical setting. All of these may be points for intervention in the course of CPP.

As Kazak et al. (2006) emphasize, potentially traumatic events related to medical trauma are nonlinear and may be recurrent or cyclical. The CPP model works to establish the caregiver as a protective shield in situations of ongoing medical stress and enhances the caregiver and child's capacities for dyadic regulation in order to optimize functioning and coping in the face of ongoing medical stressors. Interventions that help re-establish the parent as a protective shield early in the child's medical journey, possibly even occurring while the child is in the hospital, can help change the trajectory at a critical period when exposure to a medical trauma is first identified. Although the potentially traumatizing medical events may be ongoing, CPP works to prevent further traumatization from future adverse medical events by strengthening the parentchild relationship and re-establishing the parent as a source of support during future medical events.

\section{THERAPEUTIC ASSESSMENT AS A TOOL IN CPP WITH CHILDREN WITH DEVELOPMENTAL DISABILITIES}

The Assessment and Engagement phase of CPP is important with all families, but may be particularly important when working with children with developmental disabilities. The clinician needs to spend time understanding the child's diagnosis, functioning in different developmental domains, and developmental trajectory, to help guide goals and strategies for the intervention. Language, daily living skills, cognitive level, and socialization skills have been linked with positive treatment outcomes in children with autism (Sallows \& Graupner, 2005). Information from standardized developmental assessment can aid the clinician in developing appropriate goals and in choosing appropriate play materials. Incorporating a discussion with the parent regarding their child's developmental functioning helps the clinician to gain insight into the 
parent's understanding of the child's diagnosis, parental expectations and attributions, and to assess parental stress. As Ghosh Ippen et al. (2014) discussed, parental distress and grief, if not resolved, can negatively impact parents' attunement and responsiveness in their relationship with their child.

In addition to assessing the child's level of developmental functioning, more comprehensive assessment is sometimes needed to assist the therapist and the family in differentiating between symptoms explained by trauma and those associated with developmental disability. Such assessment can also help the family to understand how trauma can interefere with the expression of a child's developmental potential, beyond what is attributable to the disability itself. In the case of Juan, his father and aunt expressed confusion about the diagnosis of autism (established when Juan was $2 \frac{1}{2}$ years old) versus their growing understanding of the impact of trauma on his behaviors. More comprehensive psychological assessment was completed to confirm the diagnosis of autism and to more clearly differentiate how the autism symptoms interacted with the trauma-related symptoms.

When discussing with parents these challenging areas (e.g., the child's functioning, the diagnosis, and the parents' responses), the therapeutic assessment approach illustrated in the work of Finn, Tharinger, and their colleagues (e.g., Tharinger, Finn, Hersh, Wilkinson, Christopher, \& Tran, 2008) provides insight and guidelines for clinicians regarding how to effectively involve parents in the assessment process. Many parents of children with disabilities have had negative experiences in previous assessment encounters. A collaborative approach to engaging parents in the process of developing assessment questions, encouraging parents to be present to observe their child's strengths and difficulties during standardized assessment, incorporating parents' insights into understanding their child's performance, and soliciting their feelings about the process, all enable parents to become active, engaged participants in the assessment process. The approach to assessment feedback outlined by Tharinger et al. (2008) helps the clinician to show compassion and incorporate a parents' current story and understanding of their child, while gently challenging some of the parents' attributions.

\section{INTERSECTION OF CPP AND OTHER SERVICE SYSTEMS}

As outlined by Ghosh Ippen et al (2014), the early childhood system of care for children with developmental disabilities has expanded and linkage with early intervention/Part C services is a critical component of treatment for a young child with a disability. Ghosh Ippen et al. (2014) explore the importance of trauma-informed Part C services (Gilkerson et al., 2013) given the frequent occurrence of trauma in young children involved in the early intervention system. This perspective can be broadened further by considering the inclusion of a range of infant-family mental health principles (Heffron, 2000) within the early intervention system, and the need to expand perspectives of service delivery to incorporate these components. In particular, the focus on relationship-based approaches is a key element of infant-family mental health with clear applications to early intervention (Gilkerson \& Taylor Ritzler, 2005). A longitudinal study following 183 children with developmental disabilities from entry into early intervention services through age 10 years found that positive and developmentally-enhancing mother-infant 
interactions predicted later cognitive, communication, and social skills improvements (HauserCram et al., 2001).

While the development of the Individual Family Service Plan within the early intervention system requires the service coordinator to include the family's needs and desires within the service plan, in practice many early intervention services are provided to the child as an individual rather than through a dyadic, relationship-based approach. In our experience, the arrival of the early interventionist to the family's home often cues a period of respite for the parents rather than an opportunity to deliver dyadic developmental interventions in which the early interventionist supports the parent's interactions with the child. As illustrated in the case of James, the CPP therapist can play a key role in encouraging relationship-based, dyadic early intervention services by providing psychoeducation about infant-family mental health perspectives, working directly with the early intervention therapist to support the inclusion of parents in the intervention process, and empowering parents to take an active role in planning and delivering early interventions.

As Ghosh Ippen et al (2014) highlight, therapists trained in CPP at Children’s Hospital Los Angeles have the opportunity to work collaboratively with the interdisciplinary medical personnel, including medical social workers, nurses, doctors, occupational therapists, physical therapists, and child life specialists to provide coordinated, comprehensive care to children with special healthcare needs and their families. As in the case of James, it is often these health care providers who identify children and families who are at risk and refer the families to our mental health programs. Although James was referred in the course of medical follow-up after discharge from the hospital, a referral for CPP may be made while the infant or young child is hospitalized so treatment can begin on an inpatient medical unit. As one can imagine, the medical milieu is a unique environment to begin CPP, but it allows critical opportunities to engage with the family while they are in the midst of a medical crisis. When working in this milieu, there may be active medical crises that occur and the first step is to ensure a sense of safety and stabilization for the family. Supporting the parent-child dyad through the hospitalization process works to re-establish a sense of safety, which is one of the basic tenets of CPP.

When a family is coping with a young child's illness, the child's medical providers are an essential part of the child's system of care. Therapists may find it helpful to attend outpatient medical appointments with families, especially those families who are coping with the chronic stressor of their child's medical condition and/or a diagnosis of a developmental disability.

In the case of James, his mother shared with the therapist her insight regarding her emotional state when she experienced "trauma triggers" or reminders of James' medical trauma on a weekly basis as she and James attended his medical appointments. The therapist identified this insight as a "port of entry," and a space from which growth could occur. One possibility that would allow direct access to this port of entry would be for a therapist to attend weekly outpatient medical appointments with James and his mother in order to assist with dyadic regulation in those instances of distress. Being present in the medical setting would allow the therapist to provide support to James and his mother as they grow together to cope with the trauma triggers in the hospital setting and begin to make meaning of his medical diagnoses and 
their experiences in the medical milieu. Being present in the medical setting where trauma triggers exist allows the therapist to explore the family's understanding of their child's behavior in that setting. Similarly, participation in service planning for a child with developmental disabilities, such as meetings with early intervention or school providers, can provide opportunities for support and therapeutic intervention to address trauma triggers in parents that may be elicited in such meetings.

The presence of a therapist trained in CPP within the medical setting not only allows for support of the family and coordination of care, but it also provides an opportunity for dissemination of trauma-informed principles to interdisciplinary providers in the medical setting. When discussing how to create trauma-informed systems of care, Ko et al. (2008) emphasized the importance of the child welfare, education, first responder, juvenile justice, and health care systems working together to support children. They recommendeed trauma-informed approaches such as screening children for trauma exposure, providing education about trauma to families, and ensuring continuity of care across systems. Consultation between the CPP therapist and the child's medical team allows education of medical providers regarding trauma-informed intervention, as well as the assessment and identification of early symptoms of pediatric medical traumatic stress. These consultations provide an opportunity to encourage other providers to understand the meaning of a child's behavior in a medical setting and to consider the impact of trauma triggers on the young child's regulation and behavior in that setting.

The National Child Traumatic Stress Network (NCTSN; 2004) developed the Pediatric Medical Traumatic Stress Toolkit for Health Care Providers to assist medical providers in assessing symptoms of medical traumatic stress in pediatric patients, as well as to provide educational materials that can be distributed to families to help the family support their child through this experience. A Health Care Toolbox (Kassam-Adams, Schneider, \& Kazak, 2010) can also be accessed on-line with training and resource materials for a wide variety of providers, ranging from medical providers to child welfare providers. This website also summarizes resources for interdisciplinary training on trauma-informed principles in a medical setting. Through implementation of tools such as these in addition to direct consultation with pediatric psychologists and early childhood psychologists trained in CPP, the medical system and health care personnel are included as partners when providing trauma-informed care for young children who have been impacted by medical trauma.

\section{ADDRESSING BARRIERS TO ACCESSING SERVICE SYSTEMS FOR ETHNIC MINORITY FAMILIES OF CHILDREN WITH DISABILITIES}

Latinos and other ethnic minority populations have historically experienced gaps in accessing mental health services, and such disparities continue to the present day. Factors contributing to this gap may include low educational and economic status, discrimination, illegal immigration status, language barriers, lack of ethnically diverse mental health care providers, absence of health insurance, religious beliefs, acculturation, and beliefs about mental illness and treatment (Rios-Ellis et al., 2005; Ruiz, 2002; Ruiz, 2005). Barriers and disparities also exist in accessing early intervention services for children with developmental disabilities. Zimbardo (2011) documented in a Los Angeles Times article that expenditures by the California 
Department of Developmental Services on interventions for autism in children aged 3 to 6 were significantly lower for Latino and Black children than for White and Asian children.

The process of initiating connection to early intervention and mental health services in the cases of Juan and James illustrates barriers as well as strategies that may benefit families from ethnic minority backgrounds in accessing services. In the case of James, the medical social worker had referred him for early intervention services, but the family had not followed through with the referral and had not begun early intervention services at the time that mental health treatment began. Multiple factors may have contributed to the family's difficulty with linkage. First, language barriers have been found to be a barrier in monolingual Spanish-speaking families' access to services for their young child (Williams, Perrigo, Banda, Matic, \& Goldfarb, 2013). Second, the family was overwhelmed with caring for James' medical needs and appointments, and expressed confusion about what the early intervention services would provide, why the services might be helpful, and how to access them. The CPP therapist assisted in linkage by providing education about the way the service system was structured, and the differences in services provided by the medical team, the mental health therapist, and the early intervention system. Contacting the early intervention provider together with the parent and being present for initial services provided in the home also helped to create a bridge.

In the case of Juan, he was diagnosed with autism at age 2 years, 7 months, but had not received any early intervention or special education services through school nor through the developmental disabilities system at the time he began CPP at age 6 years. Despite the diagnosis of autism from the State developmental disabilities program, Juan had been found not eligible for special education when evaluated by the school district at age 2 years, 11 months. At the time CPP started, Juan was 6 years old, in first grade in a regular education classroom at a charter school, and he did not have an Individualized Education Plan (IEP) nor any special education services at school. His father was unaware of special education services for children with autism and the school had not initiated an IEP despite his behavioral problems and delayed academic performance in the classroom; instead, the school was recommending having him repeat first grade.

The CPP therapist played a critical role in linking the family with school services. She visited the classroom to observe Juan, meet with his teacher, and determine whether his needs were being met within the regular education setting. After discussing her concerns about Juan's difficulties in the classroom, she educated Juan's father about the IEP process, helped him to request an evaluation, and participated in the IEP meeting together with Juan's father. The school assessment led to the school developing a behavior intervention plan. Juan's father became actively involved in the school, volunteering in the classroom and appropriately advocating for his son's needs in future school planning meetings. 


\section{RECOMMENDATIONS FOR TRAINING CPP THERAPISTS AND MONITORING TREATMENT FIDELITY IN APPLICATIONS TO YOUNG CHILDREN WITH DEVELOPMENTAL DISABILITY}

While CPP has promise as an intervention approach for young children with developmental disabilities, it is important that CPP therapists working with this population receive enhanced training to ensure they have the necessary knowledge and skills to meet the unique needs of children with disabilities. Specific recommendations for domains of training include: (1) knowledge about developmental disabilities including symptoms, etiology, and impact on behavioral and emotional functioning; (2) knowledge about the impact of pediatric medical traumatic stress on young children and their families; (3) knowledge about the systems of care and services available for young children with developmental disabilities, including Part $\mathrm{C}$ early intervention services, preschool special education, and funding to support families caring for a child with a disability; (4) skill in supporting families to navigate service systems and knowledge about legal advocacy issues such as rights related to timelines, determination of eligibility, and development of service or education plans; (5) knowledge of the scope and contributions of interdisciplinary professionals who provide services to children with developmental disabilities, and skill in working collaboratively with professionals from other disciplines; and (6) skill in communicating recommended applications of a trauma-informed and relationship-based perspective to early intervention, special education, and medical service providers.

The developers of CPP have created fidelity checklists to support therapists in learning the CPP model and monitoring fidelity to its principles (Ghosh Ippen, 2012). Our work in applying the model to young children with developmental disabilities leads to recommendations for additions to the fidelity measures when applying the CPP model to this population. In the Assessment and Engagement phase, it is recommended that the CPP therapist (1) assess the parents' understanding of the child's diagnosis of developmental disability, attributions about the likely cause of the diagnosis, level of acceptance of the diagnosis, and the family's degree of hope for long-term positive outcomes for the child and family in the context of the diagnosis; (2) assess the child's developmental functioning in key domains (e.g. cognitive, language, motor, social-emotional) and share information with the family about the child's level of functioning, strengths, and intervention needs in therapeutic and collaborative ways; (3) obtain information about the child's connection with early intervention or special education services and supports, provide education to the family about the service delivery system, and support the family in navigating the service system; and (4) with the family's permission, communicate with medical personnel, early intervention therapists, and school personnel to ensure that services are coordinated and to provide a trauma-informed and relationship-based perspective to other providers working with the child and family.

When assessing Intervention content fidelity, it is recommended that the therapist ensure that the selection of toys for sessions and the implementation of interventions are appropriate to the child's developmental level. The CPP goal of "returning to a normal developmental trajectory" should be modified to focus on supporting the child in reaching their developmental 
potential and maximizing functioning and quality of life in the context of constraints related to the developmental disability.

An additional domain of CPP intervention important to work with children with developmental disabilities is collaboration with the early intervention and/or special education service system and with medical professionals. Fidelity monitoring in this area may include (1) documenting contact with professionals providing other services to the child; (2) consistent with dyadic-relational fidelity, providing consultation to service providers about dyadic approaches to intervention and encouraging parents to actively participate in services provided by other professionals; (3) consistent with reflective practice fidelity, the clinician reflects on her own reactions and responses to other service providers and seeks consultation to develop effective and collaborative approaches to resolving any differences with other providers; (4) consistent with trauma framework fidelity, the therapist provides consultation to other providers about the impact of trauma on the child's symptoms and behaviors and ways to provide intervention in trauma-informed ways, and/or supports the parent in sharing the trauma-informed perspective with other providers. Finally, when considering fidelity monitoring related to the CPP therapist's reflective practice and participation in supervision, it is recommended to incorporate the clinician's reflections regarding working with a child with a disability, the clinician's views about the meaning of disability and the role of individuals with disability in society and within the family, and any differences that may arise between the clinician's and the family's attitudes about and perspectives on disability.

\section{REFERENCES}

Bayat, M. (2007). Evidence of resilience in families of children with autism. Journal of Intellectual Disability Research, 51, 702-714.

Center for Pediatric Traumatic Stress (n.d.). Medical events and traumatic stress in children and families. Retrieved November 3, 2014 from http://www.nctsn.org/sites/default/files/assets/pdfs/MedicalTraumaticStress.pdf.

Chamak, B., Bonniau, B., Oudaya, L., \& Ehrenberg, A. (2011). The autism diagnostic experiences of French parents. Autism, 15, 83-97.

Emerson, E. (2003). Mothers of children and adolescents with intellectual disability: Social and economic situation, mental health status, and the self-assessed social and psychological impact of the child's difficulties. Journal of Intellectual Disability Research, 47, 385399.

Ferguson, P. M. (2002). A place in the family: An historical interpretation of research on parental reactions to having a child with a disability. Journal of Special Education, 36, $124-130$.

Ghosh Ippen, C. (2012). Child-Parent Psychotherapy Fidelity Checklists. Unpublished manuscript.

Ghosh Ippen, C., Noroña, C. R., \& Lieberman, A. F. (2014). Clinical considerations for conducting Child-Parent Psychotherapy with young children with developmental disabilities who have experienced trauma. Pragmatic Case Studies in Psychotherapy, 10(3), Article 2, 196-211. Available: http://hdl.rutgers.edu/1782.1/pcsp_journal 
Gilkerson, L., Graham, M., Harris, D., Oser, C., Clarke, J., Hairston-Fuller, T., \& Lertora, J. (2013). Trauma-Informed Part C early intervention: A vision, a challenge, a new reality. Zero to Three , 34, 34-44.

Gilkerson, L. \& Taylor Ritzler, T. (2005). The role of reflective process in infusing relationshipbased practice into an early intervention system. In K.M. Finello (Ed.), The handbook of training and practice in infant and preschool mental health. New York: John Wiley \& Sons.

Green, S. E. (2007). "We're tired, not sad”: Benefits and burdens of mothering a child with a disability. Social Science and Medicine, 64, 150-163.

Harley, E.K., Williams, M.E., Zamora, I., \& Lakatos, P.P. (2014). Trauma treatment in young children with developmental disabilities. Applications of the Child-Parent Psychotherapy (CPP) Model in the Cases of "James" and “Juan”. Pragmatic Case Studies in Psychotherapy,10(3), Article 1, 156-195. Available: http://hdl.rutgers.edu/1782.1/pcsp_journal

Hauser-Cram, P., Erickson Warfield, M., Shonkoff, J. P., Wyngaarden Krauss, M., Sayer, A., Christofk Upshur, C., \& Hodapp, R. M. (2001). Children with disabilities: A longitudinal study of child development and parent well-being. Monographs of the Society for Research in Child Development, 66(3), 1-126.

Hedov, G., Wikblad, K., \& Anneren, G. (2007). First information and support provided to parents of children with Down syndrome in Sweden: Clinical goals and parental experiences. Acta Pediatrica, 91, 1344-1349.

Heffron, M. (2000). Clarifying concepts of infant mental health-Promotion, relationship-based preventive intervention, and treatment. Infants and Young Children, 12, $14-21$.

Kassam-Adams, N. \& Fein, J. (2003). Posttraumatic stress disorder and injury. Clinical Pediatric Emergency Medicine, 4, 148-155.

Kassam-Adams, N., Schneider, S., \& Kazak, A. E. (2010). Health Care Toolbox: Your guide to helping children and families cope with illness and injury. Retrieved October 29, 2014, from http://www.healthcaretoolbox.org/

Kazak, A. E., Kassam-Adams, N., Schneider, S., Zelikovsky, N., Alderfer, M. A., Rourke, M. (2006). An integrative model of pediatric medical traumatic stress. Journal of Pediatric Psychology, 31, 343-355. 2005 doi:10.1093/jpepsy/jsj054

Kazak, A. E., Stuber, M. L., Barakat, L. P. , Meeske, K., Guthrie, D., \& Meadows, A. T. (1998). Predicting posttraumatic stress symptoms in mothers and fathers of survivors of childhood cancers. Journal of the American Academy of Child \& Adolescent Psychiatry, 37, 823-831. doi: 10.1097/00004583-199808000-00012

Kerr, S. M. \& McIntosh, J. B. (2000). Coping when a child has a disability: Exploring the impact of parent-to-parent support. Child Care Health Delivery, 26, 309-322.

Ko, S. J., Ford, J. D., Kassam-Adams, N., Berkowitz, S. J., Wilson, C., Brymer, M. J., \& Layne, C. M. (2008). Creating trauma-informed systems: child welfare, education, first responders, health care, juvenile justice. Professional Psychology Research and Practice, 39, 396-404. doi:10.1037/0735-7028.39.4.396

Landolt, M. A., Vollrath, M., Ribi, K., Gnehm, H. E., \& Sennhauser, F. H. (2003). Incidence and associations of parental and child posttraumatic stress symptoms in pediatric patients. Journal of Child Psychology and Psychiatry, 44, 1199-1207. doi: 10.1111/14697610.00201 
Law, M., King, S., Stewart, D., \& King, G. (2001). The perceived effects of parent-led support groups for parents of children with disabilities. Physical and Occupational Therapy in Pediatrics, 21, 29-48.

Mandell, D.S. \& Salzer, M. S. (2007). Who joins support groups among parents of children with autism? Autism, 11, 111-122.

National Child Traumatic Stress Network. (2004). Pediatric medical traumatic stress toolkit for health care providers. Retrieved October 30, 2014, from http://www.nctsn.org/traumatypes/pediatric-medical-traumatic-stress-toolkit-for-health-care-providers.

O’Brien, M. \& Daggett, J. A. (2006). Beyond the autism diagnosis: A professional's guide to helping families. Baltimore, MD: Brookes Publishing Company.

Rios-Ellis, B., Aguilar-Gaxiola, S., Cabassa, L., Caetano, R., Comas-Diaz, L., Flores, Y., Gonzalez, H., Lopez, S., Lopez-Zetina, J., Rodriguez Chandler, L., Leon, R., \& Ugarte, C. (2005). Critical disparities in Latino mental health: Transforming research into action. Retrieved from www.nclr.org.

Ruiz, P. (2002). Hispanic access to health/mental health services. Psychiatric Quarterly, 73, 85-91.

Ruiz, P. (2005). Hispanics' mental healthcare plight. Behavioral Health Management, 25, 17-19.

Sallows, G. O., \& Graupner, T. D. (2005). Intensive behavioral treatment for children with autism: Four-year outcome and predictors. American Journal on Mental Retardation, 110, 417-438. doi:http://dx.doi.org/10.1352/0895-017(2005)110[417:IBTFCW]2.0.CO;2

Saxe, G., Vanderbilt, D., \& Zuckerman, B. (2003). Traumatic stress in injured and ill children. PTSD Research Quarterly, 14, 1-7.

Scorgie, K. \& Sobsey, D. (2000). Transformational outcomes associated with parenting children who have disabilities. Mental Retardation, 38, 195-206.

Scorgie, K. \& Wilgosh, L. (2008). Reflections on an uncommon journey: A follow-up study of life management of six mothers of children with diverse disabilities. International Journal of Special Education, 23, 103-114.

Scorgie, K., Wilgosh, L., \& Sobsey, D. (2004). The experience of transformation in parents of children with disabilities: Theoretical considerations. Developmental Disabilities Bulletin, 32, 84-110.

Shakespeare, T., Iezzoni, L. I. \& Groce, N. E. (2009). Disability and the training of health professionals. The Lancet, 374, 1815-1816.

Solomon, M., Pistrang, N., \& Barker, C. (2001). The benefits of mutual support groups for parents of children with disabilities. American Journal of Community Psychology, 29, 113-132.

Stanton, T. \& Besser, H. (1998). The positive impact of children with an intellectual disability on the family. Journal of Intellectual and Developmental Disability, 23, 57-70.

Stuber, M., Shemesh, E., \& Saxe, G. (2003). Posttraumatic stress responses in children with lifethreatening illness. Child and Adolescent Psychiatric Clinics of North America, 12, 195209.

Taunt, H. M. \& Hastings, R. P. (2002). Positive impact of children with developmental disabilities on their families: A preliminary study. Education and Training in Mental Retardation and Developmental Disabilities, 37, 410-420.

Tharinger, D. J., Finn, S. E., Hersh, B., Wilkinson, A., Christopher, G. B., \& Tran, A. (2008). Assessment feedback with parents and preadolescent children: A collaborative approach. Professional Psychology: Research and Practice, 39, 600-609. 
Williams, M. E., Perrigo, J., Banda, T., Matic, T., \& Goldfarb, F. (2013). Barriers to accessing services for young children. Journal of Early Intervention, 35, 61-74.

Young, G. S., Mintzer, L. L., Seacord, D., Castañeda, M., Mesrkhani, V., \& Stuber, M. (2003). Symptoms of posttraumatic stress disorder in parents of transplant recipients: Incidence, severity, and related factors. Pediatrics, 111(6), e725-e731.

Zimbardo, A. (2011). Warrior parents fare best in securing autism services. Retrieved November 2, 2014 from www.latimes.com 